\title{
Design of a quasi-passive 3 DOFs ankle-foot wearable rehabilitation orthosis
}

\author{
Chao Zhang ${ }^{\mathrm{a}}$, Yanhe Zhu ${ }^{\mathrm{a},{ }^{*}}$, Jizhuang Fan ${ }^{\mathrm{a}}$, Jie Zhao ${ }^{\mathrm{a}}$ and Hongying $\mathrm{Yu}^{\mathrm{b}}$ \\ ${ }^{a}$ State Key Laboratory of Robotics Institute, Harbin Institute of Technology, Harbin, 150006, \\ Heilongjiang, China \\ ${ }^{b}$ School of Mechatronics Engineering, Harbin Institute of Technology, Harbin, 150001, Heilongjiang, \\ China
}

\begin{abstract}
Muscular rigidity and atrophy caused by long-term underactivity usually lead to foot drop, strephenopodia, foot extorsion or some other complications for the lower limb movement disorders or lower limb surgery sufferers. The ankle-foot orthosis can help patients conduct the right ankle motion mode training, inhibit spasm and prevent ankle complications. In this paper, a quasi-passive 3 DOFs ankle-foot wearable orthosis was designed on the basis of kinematics and dynamics analysis of the ankle joint. Ankle joint trajectory and dynamic characteristics similar to those of natural gait can be obtained by the combination of passive energy storage and additional power complement. In terms of function, the orthosis has shock absorption and low energy consumption. Given its excellent characteristics of comfortableness, lightweight, and anthropomorphic construction, the orthosis can be used in medical institutions for rehabilitation training or as a daily-walking auxiliary equipment for surgery sufferers.
\end{abstract}

Keywords: Ankle-foot wearable orthosis, rehabilitation, quasi-passive, anthropomorphic design

\section{Introduction}

Foot drop, strephenopodia, and foot extorsion are common complications of lower limb movement disorders (hemiplegia and paraplegia) and lower limb surgery. These complications usually manifest as abnormal gait and require utilization of an orthosis or a crutch. A proper ankle-foot orthosis can expand the contact area between the foot and the ground, stabilize the ankle joint, and correct the foot deformity. As a result, the abnormal movement patterns could be curbed for the improvement of motion coordination and the recovery of motor functions [1]. Traditional ankle-foot orthoses generally assume a half-surrounded configuration from the posterior shank to the planta to fix and inhibit ankle distortion. These orthoses can be broadly separated into two categories: protective and supportive. The protective type is not suitable for walking because of its integrated molding design; thus, it can only serve for prevention and correction. Meanwhile, the posterior shank section and the planta section of the supportive type are connected by a hinge or an elastic rebound sheet for the flexion/extension

\footnotetext{
* Address for correspondence: Yanhe Zhu, State Key Laboratory of Robotic and System, Harbin Institute of Technology, Building C1-203, HIT Science Park, Yikuang Street No. 2, Nangang District, 150006 Harbin, P.R. China. Tel.: 13074594165; Fax: +86-045186414538; E-mail: yhzhu@hit.edu.cn.
} 
rotation of the ankle. Clinical studies have shown that hinged ankle-foot orthoses elicit better recovery effects than normal orthoses $[2,3]$.

In the last decade, considerable research has focused on powered orthoses, which exhibit obvious advantages compared with traditional orthoses $[4,5]$. The treatment of foot lesion deformity mainly includes three phases. In the early phase, passive movement is proceeded by a therapist to maintain muscle activity. In the middle phase, balance training exercises such as wobble board exercises are adopted to help patients acquire self-balance. In the late phase, muscle strength training is conducted to realize natural human gait. Powered ankle-foot rehabilitation devices are divided into two main types according to the appropriate treatment stage: fixed ankle-foot rehabilitation training platform and wearable rehabilitation orthosis.

Fixed ankle-foot rehabilitation training platforms are suitable for the early and middle phases of rehabilitation training. Y. Jungwon [6] designed a novel reconfigurable ankle-foot rehabilitation robot for ankle rehabilitation training. The four DOFs formed by the combination of two parallel mechanisms are driven by air cylinders to implement ankle flexion/extension, abduction/adduction, internal/external rotation, and forefoot rotation. Another dual Stewart platform for bipedal rehabilitation training developed by Rutgers University has 12 DOFs that are driven by air cylinders on the basis of pneumatic servo control [7]. Therapists can remotely mentor training through the realtime reproduction of virtual movements of the network. However, it is limited by the mechanism complexity and the requirement of independent air supply. Wearable rehabilitation orthoses are appropriate for middle and later periods of rehabilitation training. Well-known orthoses of this type include the variable-impedance ankle-foot orthosis developed at MIT [8], the ankle-foot orthosis for stroke rehabilitation by Florida International University [9], and the ankle-foot orthosis using artificial pneumatic muscles designed by Gordon [10]. Both the first two are hinged foot-ankle exoskeleton orthosis powered by series elastic actuator.

The 3 DOFs ankle-foot rehabilitation orthosis presented in this paper is for ground walking exercise in the middle and late phases of rehabilitation training. The wearer would accomplish normal ankle movement with the help of an auxiliary torque provided by the passive energy storage device and the motor. The ankle is a flexible joint, and practical requirements such as comfortable and simple wearing method, lightweight, low energy consumption, and high bionic performance are considered in the design process of the orthosis.

\section{Biomechanical analysis and system design goals}

Rigorous analysis of the ankle kinematics and dynamics performance in ground walking is crucial to understand the ankle motion mechanism, the configuration scheme setting, and the choice of lowenergy-consumption method. The changes in joint angle and torque are acquired and then used to calculate the angular velocity and energy variation, which are the basis of the system design.

The ankle joint can be approximately simplified as a sphere-pin pair including three DOFs as shown in Figure 1: flexion/extension, abduction/adduction, and internal/external rotations. Ankle-foot wearable orthoses serve as the physical interface of human-computer interaction. As a result, the comfortableness and practicality of the process should be the primary considerations. Thus, the orthosis design must follow the anthropomorphic principle.

Since we intended to design an anthropomorphic orthosis, the required rotation ranges and torques for each DOF of the ankle joint should be close to those required by natural human walking. In addition, the primary goal of a rehabilitation orthosis is to assist locomotion. Hence, the power 


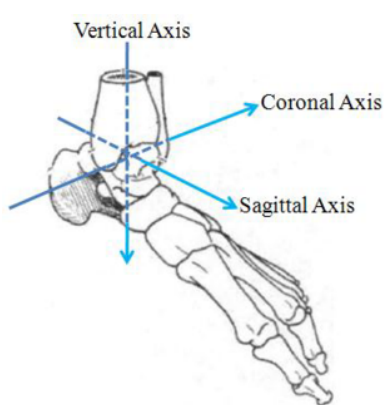

Fig. 1. The 3 DOF of ankle joint.

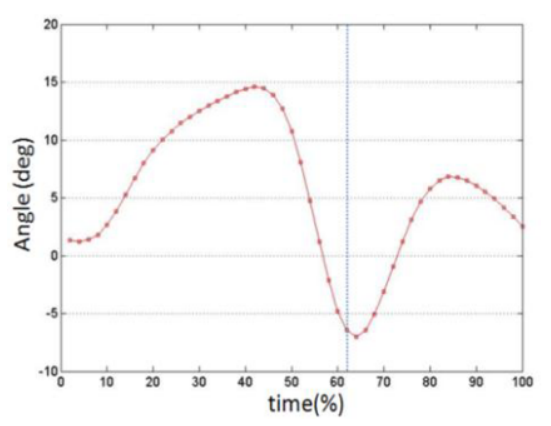

Fig. 2. Ankle flexion/extension angle.

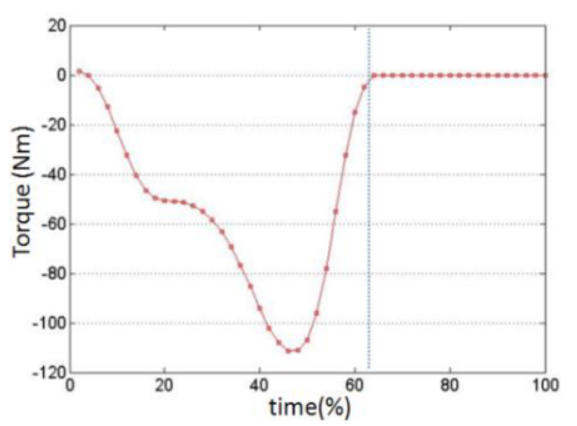

Fig. 3. Ankle flexion/extension torque.

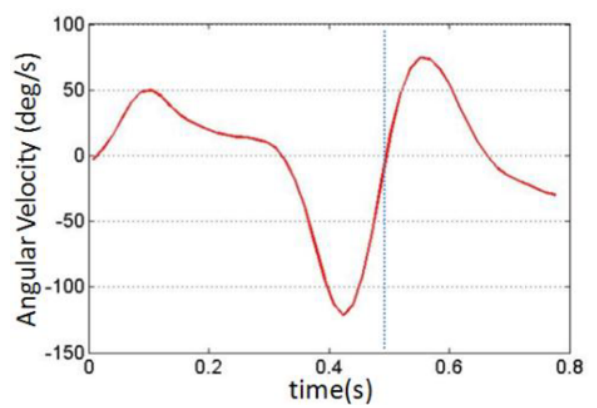

Fig. 4. Angular velocity variation of the ankle flexion/ extension DOF.

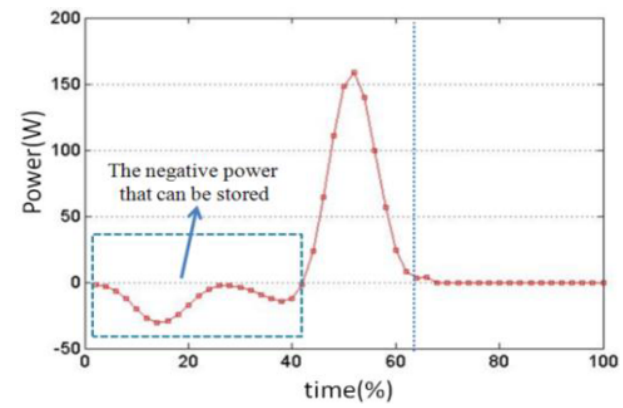

Fig. 5. Ankle flexion/extension instantaneous mechanical power.

consumption should also be estimated by analyzing the walking kinematics and dynamics performance. As a professional human body biomechanics simulation software, LifeMod has been widely used in human movement simulation for its diverse functions and accuracy $[11,12]$. During the biomechanical analysis in this study, a 20-year-old male simulation model with a height of $175 \mathrm{~cm}$ and a weight of 70 $\mathrm{kg}$ was built in accordance with the "GB 10000-1988 Chinese adults body size" issued by the China National Standardization Management Committee. The ankle flexion/extension angle and torque curves at a walking speed of $1.0 \mathrm{~m} / \mathrm{s}$ are obtained by the simulation as shown in Figures 2 and 3, respectively. The vertical curve is the boundary between the stand and swing phases. The simulation result indicates that the main motion mode is the flexion/extension rotation accompanied by minimal motions of the other two DOFs for a harmonious and natural gait. In the sagittal plane, the rotation range is approximately $22^{\circ}$, including a maximum flexion angle of $15^{\circ}$ in the late stance phase and a maximum extension angle of $-7^{\circ}$ after the toe-off moment. The ankle torque is almost entirely negative during the stand phase and approximately zero during the swing phase. The joint angular velocity is obtained by the angle curve differential, which is then used to calculate the power curve (Figures 4 and $5)$.

The maximum instantaneous angular velocity and power are $120 \%$ and $160 \mathrm{~W}$. Both the two indexes should be attained in the ankle-foot wearable orthosis design. The goal of this paper is to seek a wearable orthosis designing approach that is capable to provide a humanlike kinematics and dynamics performance while still matching the biological shape in a convenient and comfortable wearing method. In specific, the ankle-foot orthosis should satisfy the following design goals:

a) Anthropomorphic configuration to entirely fit human ankle motion space;

b) Necessary balance elastic support in all three DOFs to inhibit foot drop, strephenopodia, and 
foot extorsion;

c) Reasonable energy storage in the dotted box (i.e. negative power) as shown in Figure 5 and energy release capacity during the positive power phase. The net energy consumption will be complemented by external drive such as a motor;

d) Mimicking capacity for the dynamics of the human ankle, representing that the maximum angular velocity, torque, and power provided by the orthosis should meet the minimum requirements as of $120 \%, 120 \mathrm{Nm}$, and $160 \mathrm{~W}$ on the flexion/extension degree of freedom;

e) Reasonable information detection methods to judge the motion state of human foot, which will enable the orthosis to provide appropriate auxiliary force in a timely manner;

f) Lighter weight and convenient wearing method.

\section{Energy economy optimization}

As a mobile walk training equipment, the ankle-foot orthosis should be powered by lithium batteries to operate for a relatively long time. Thus, building an energy-economic and practical ankle-foot orthosis is challenging. The analysis in the previous section demonstrates the possibility of a passive energy storage throughout each early stance period. However, the average ankle power is positive, especially in the late stance period, which requires a high instantaneous power output to propel the wearer. Therefore, a concrete implementation scheme of passive energy storage and additional active power complement is illustrated in this section for the selection of a reasonable spring stiffness and an appropriate motor power. An effective combination of the two features is important to reduce energy consumption.

The solid line in Figure 6 shows the ankle torque-angle behavior during a walking cycle on the basis of the simulation data, and the dashed line is an approximate fitting for analysis convenience. Several significant phases in walking are graphically expressed in Figure 7. Points (1) and (2) denote the gait events of heel strike and foot flat respectively. The maximum dorsiflexion occurs at the heel-off moment between points (3) and (4), and then segment (5)-(6) represents a smooth toe-support phase with a linear decrease in the ankle torque. Segments (6)-(7) and (7)-(1) represent the swing phase with a natural dorsiflexion and plantar flexion. The dashed line from points (2) to (3) shows the negative torque that can be utilized by installing a spring structure, and its slope represents the spring stiffness that should be designed. The area enclosed by the dashed lines is the network accomplished by the motor throughout each late stance period with an energy value of approximately14.22 J. The negative

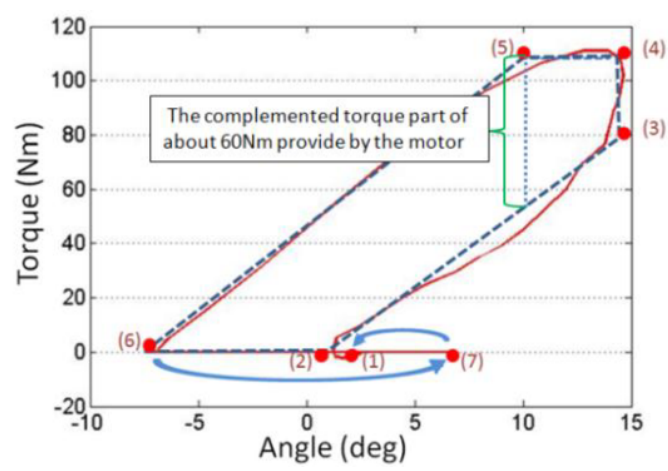

Fig. 6. Ankle torque versus angle.

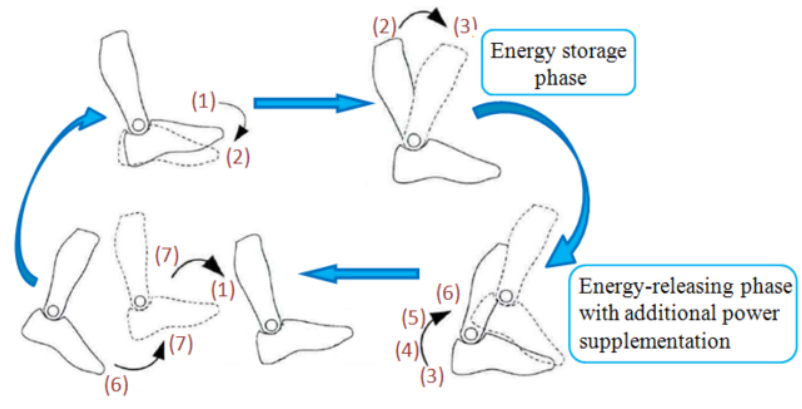

Fig. 7. Summary of significant phases in walking. 


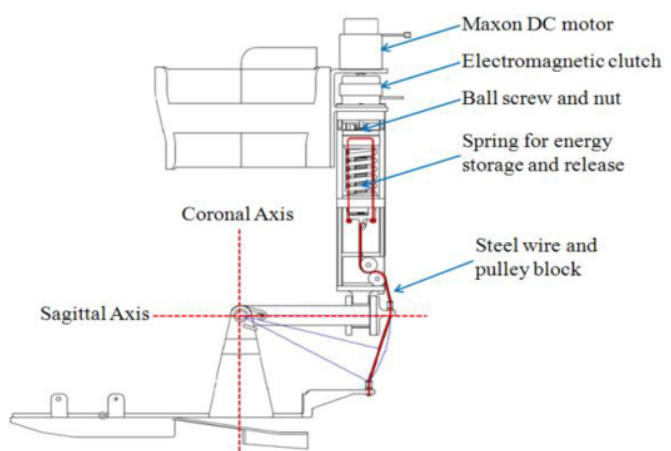

Fig. 8. The force transmission method using steel wire.

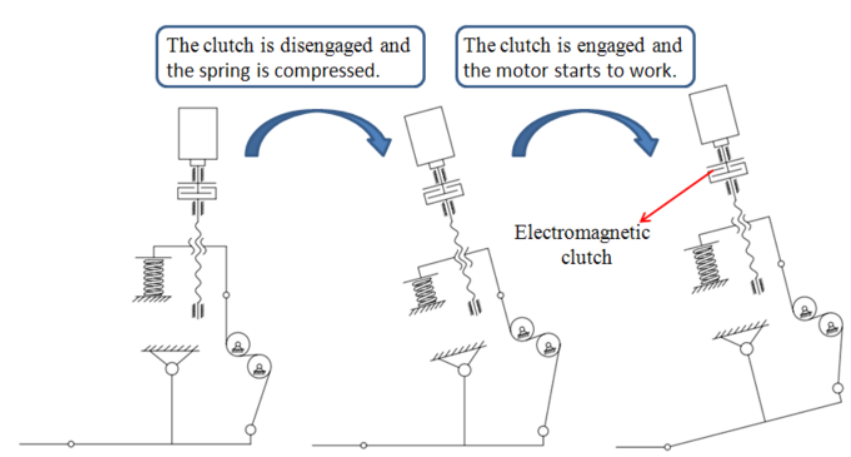

Fig. 9. Two kinds of working states for the electromagnetic clutch.

power that can be stored is the triangular area under the dashed line from points (2) to (3) with the energy of approximately $9.77 \mathrm{~J}$. Thus, the total energy required for a gait cycle is $24 \mathrm{~J}$ for a person (70 $\mathrm{kg}$ ) walking at a constant speed of $1.0 \mathrm{~m} / \mathrm{s}$. In theory, a quasi-passive ankle-foot orthosis combining passive energy storage and active driving might reduce energy consumption by $40.7 \%$. However, in actual situations, all the factors such as individual walking habits, mechanical transmission efficiency, spring energy storage efficiency, and friction loss of the rotational joint will affect energy storage capacity.

In the concrete implementation process, a novel mechanical transmission method that involves the combination of an electromagnetic clutch, ball screw, and steel wire is designed in Figure 8. The ball screw has bidirectional power transmission and small friction coefficient, indicating a high transmission efficiency of $90 \% \sim 98 \%$. In the early support phase, the electromagnetic clutch is disengaged and the spring is compressed for energy storage. In the late support phase, the electromagnetic clutch is engaged, and the required ankle joint torque is provided by the spring and the motor (Figure 9).

The energy storage spring stiffness can be approximated as

$$
k=\frac{180 \cdot \mathrm{T}_{3}}{\pi \mathrm{r}^{2}\left(\theta_{3}-\theta_{2}\right)}=17.97 \mathrm{~N} / \mathrm{mm}
$$

where $T_{3}$ is the torque of point (3); $\theta_{2}$ and $\theta_{3}$ are the angles at point (2) and (3); and $\mathrm{r}$ is the moment arm of the wire rope.

The choice of the selected motor must meet the power requirements firstly. During the motor working phase, the additional complemented torque is approximately $60 \mathrm{Nm}$, and the maximum instantaneous angular velocity is $120 \%$. As a result, the motor power should be larger than the product of the two, which is $125 \mathrm{~W}$. In consideration of enough power margin, a 150W Graphite Brushes DC motor (Maxon RE40) is selected with a nominal speed of $4920 \mathrm{rpm}$, and a nominal torque of $0.177 \mathrm{Nm}$ is selected. Furthermore, the ball screw transmission is also chosen with a ball screw lead of $4 \mathrm{~mm}$ and a maximum transmission load rating of $3.6 \mathrm{kN}$ is also selected which is sufficiently large for the power transmission.

\section{Design architecture and system implementation}


A reasonable solution to the contradiction between the anthropomorphic architecture and the interference avoidance of man-machine movement space is a challenge for the ankle-foot orthosis design. The following sections discuss the critical features in the mechanical design.

\subsection{Anthropomorphic consideration and the joint ranges determination}

The anthropomorphic architecture with a similar kinematics property to human was chosen for the ankle-foot wearable rehabilitation orthosis. Therefore, the DOF design must be entirely equal to the human body. The main ankle motion mode is the flexion/extension rotation accompanied by minimal motions of the other two DOFs to ensure moving smoothness and coordination. Existing products or experimental prototypes usually consider the identity of the flexion/extension and abduction/adduction DOF to the human ankle but ignore the rotation internal/external DOF because of man-machine movement space interference. In this paper, a double-tier beleaguered structure is proposed to solve this problem. Overall, the DOF distribution of the ankle-foot wearable rehabilitation orthosis is shown in Figure 10. For all the DOFs, Table 1 lists the rotation ranges that entirely meet the requirement of normal walking.

\subsection{Mechanical design of the quasi-passive ankle-foot orthosis}

The mechanical design mainly includes the rotation DOF implementation surrounding the shank, mechanical transmission structure based on ball screw and steel wire transmission, necessary elastic

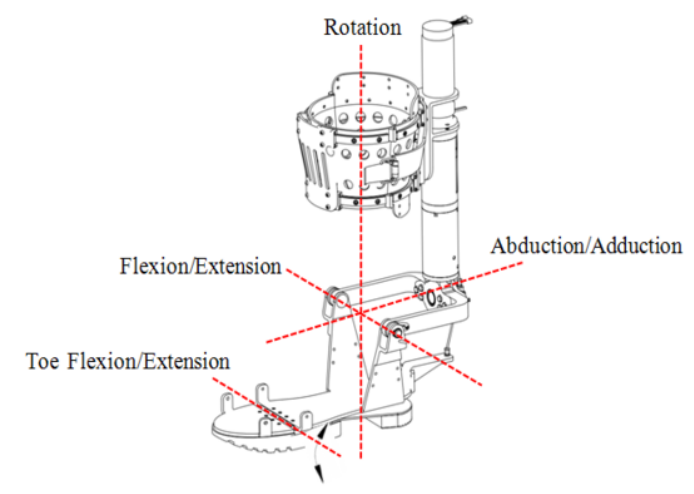

Fig. 10. Degrees of freedom for the ankle-foot orthosis design.

Table 1

Joint ranges of motion for the ankle-foot orthosis

\begin{tabular}{llll}
\hline Rotary motion & $\begin{array}{l}\text { Arthrosis } \\
\text { rotation } \\
\text { range }\end{array}$ & $\begin{array}{l}\text { Human } \\
\text { walking } \\
\text { maximum }\end{array}$ & $\begin{array}{l}\text { Ankle-foot } \\
\text { orthosis } \\
\text { maximum }\end{array}$ \\
\hline Ankle flexion & $20^{\circ}$ & $14.1^{\circ}$ & $19^{\circ}$ \\
Ankle extension & $45^{\circ}$ & $20.6^{\circ}$ & $29^{\circ}$ \\
Ankle abduction & $20^{\circ}$ & $2.1^{\circ}$ & $10^{\circ}$ \\
Ankle adduction & $30^{\circ}$ & $1.5^{\circ}$ & $10^{\circ}$ \\
Rotation external & $20^{\circ}$ & $1.6^{\circ}$ & $15^{\circ}$ \\
Rotation internal & $20^{\circ}$ & $11.5^{\circ}$ & $15^{\circ}$ \\
Toe flexion & $70^{\circ}$ & $/$ & $60^{\circ}$ \\
Toe extension & $20^{\circ}$ & $/$ & $/$ \\
\hline
\end{tabular}




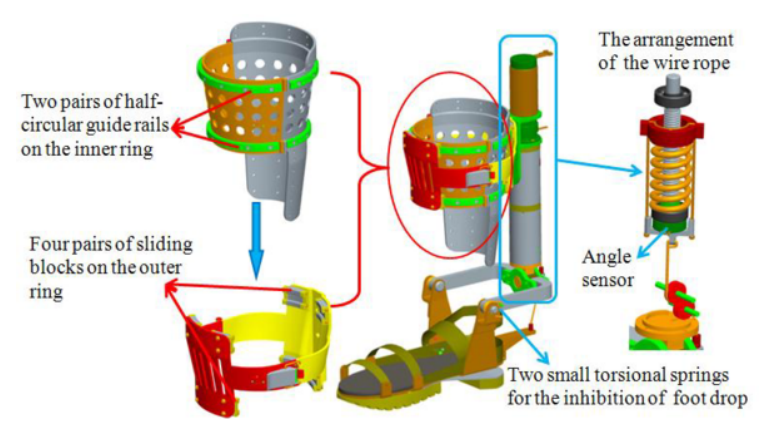

Fig. 11. Double-tier beleaguered structure design and the arrangement of the wire rope.

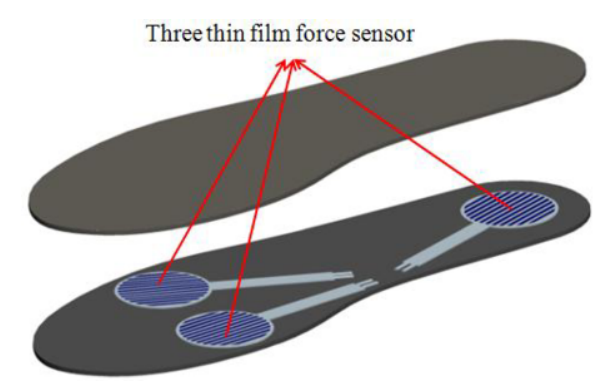

Fig. 13. Detection of the plantar pressure distribution by three thin film force sensors.

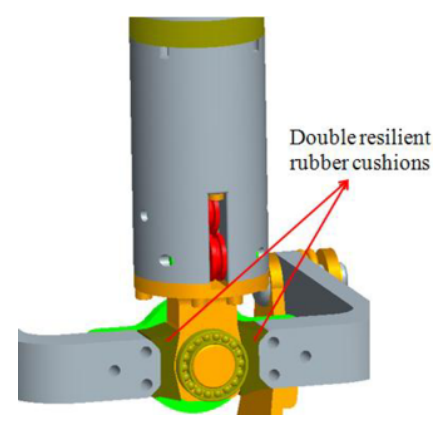

Fig. 12. Two resilient rubber cushions for the inhibition of strephenopodia and strephexopodia.

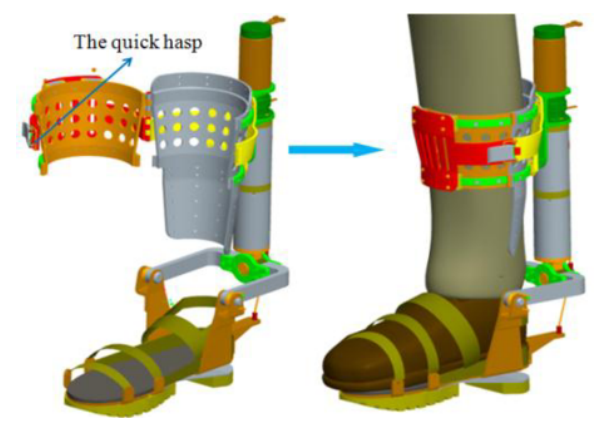

Fig. 14. Convenient wearing method by opening or closing the quick hasp.

balance support for deformity correction, and information detection of foot motion.

The double-tier beleaguered structure designed for the rotation DOF is composed of the inner and outer rings that can rotate relatively to each other as shown in Figure 11. Foot extorsion can be limited by threading rope through the lightening holes on both the inner and outer rings. Additional power com plement by the motor is transformed from rotary to linear motion by the ball screw and then used to pull the steel wire and propel the wearers along with the release of spring energy. Two small torsional springs are set for the foot drop inhibition, and a pair of resilient rubber cushions is also installed to the abduction/adduction DOF (Figure 12) for strephenopodia inhibition.

The magnetic sensitive angle sensor under the ball screw detects the rotation angle. The steel wire is tight in the entire support phase. As a result, the flexion/extension rotation angle could be derived from the ball screw rotation angle by using a simple calculation of the trigonometric function. In addition, the foot motion state in a walking gait cycle should be detected to provide appropriate auxiliary force in a timely manner. A dynamometer in sole with three thin film force sensors is designed with a position layout of one on the heel and two on the forefoot (Figure 13).

Figure 14 shows the convenient wearing method benefitting from the usage of a quick hasp for the open and shut of half the double-tier beleaguered structure. The shoe sole is composed of a $2 \mathrm{~mm}$ thick elastic steel plate and rubber pad, allowing shock absorption and raising the heel in the middle stand phase.

\section{Conclusion}


A quasi-passive three DOFs ankle-foot wearable orthosisis designed to improve ankle lesions with right ankle motion mode training. The system design goals were determined through the biomechanical analysis of the ankle joint. The system design goals were determined by the biomechanical analysis of the ankle joint. The combination of passive energy storage and additional power complement leads to an energy consumption reduction of about $40.7 \%$ in the ideal case. The convenient wearing method is also an important advantage. In our future work, the total energy consumption of the system will be experimentally validated to ensure that the orthosis can function in a relatively long time with a battery of limited weight.

\section{Acknowledgment}

The work reported in this paper is funded by "National High Technology Research and Development Program of China (863 Program)" (Grant No. 2012AA041505) and "Self-Planned Task of State Key Laboratory of Robotics and System (HIT)" (Grant No. SKLRS201201A02).

\section{References}

[1] J. Leung and A. Moseley, Impact of ankle-foot orthoses on gait and leg muscle activity in adults with hemiplegia: Systematic literature review, Physiotherapy 89 (2003), 39-55.

[2] N. Yi, B.S. Wang, L. Li, et al., Effects on ambulation of children with spastic cerebral palsy by different kind of ankle foot orthoses, Chinese Journal of Rehabilitation Medicine 17 (2002), 100-101.

[3] J. Romkes and R. Brunner, Comparison of a dynamic and a hinged ankle-foot orthosis by gait analysis in patients with hemiplegic cerebral palsy, Gait and Posture 15 (2002), 18-24.

[4] Aukje S. Andringa, Ingrid G.L. van de Port and Jan-Willem G. Meijer, Tolerance and effectiveness of a new dynamic hand-wrist orthosis in chronic stroke patients, Neuro Rehabilitation 33 (2013), 225-231.

[5] I. Yamamoto, N. Inagawa and M. Matsui, Research and development of compact wrist rehabilitation robot system, BioMedical Materials and Engineering 24 (2014), 123-128.

[6] J. Yoon and J. Ryu, A novel reconfigurable ankle/foot rehabilitation robot, Proceedings of the 2005 IEEE International Conference on Robotics and Automation, Barcelona, Spain, 2005, pp. 2290-2295.

[7] R.F. Boian, M. Bouzit, G. Burdea, J. Lewis and J.E. Deutsch, Dual Stewart platform mobility simulation, International Conference on Rehabilitation Robotics, Chicago, USA, 2005, pp. 550-555.

[8] J.A. Blaya and H. Herr, Adaptive control of a variable-impedance ankle-foot orthosis to assist drop-foot gait, IEEE Transactions on Neural Systems and Rehabilitation Engineering 12 (2004), 24-31.

[9] S.M. Chang and D. Rincon, Biofeedback controlled ankle foot orthosis for stroke rehabilitation to improve gait symmetry, Florida Conference on Recent Advances in Robotics, Miami, USA, 2006, pp. 1-5.

[10] K.E. Gordon, G.S. Sawieki and D.P. Ferris, Mechanical performance of artificial pneumatic muscles to power an anklefoot orthosis, Journal of Biomechanics 39 (2006), 1832-1841.

[11] M. Baritz, D. Cotoros, L. Cristea, et al., Assessment of human bio-behavior during gait process using LifeMod software, Broad Research in Artificial Intelligence and Neuroscience 1 (2011), 169-177.

[12] K.T. Huynh, I. Gibson, W.F. Lu, et al., Simulating dynamics of thoracolumbar spine derived from Lifemod under haptic forces, World Academy of Science, Engineering and Technology 64 (2010), 278-285. 